Canadian Journal of Higher Education Revue canadienne d'enseignement supérieur

Volume 43, No. 1, 2013, pages 44-62

\title{
L'expérience sociale des institutions scolaires : construire son identité linguistique au fil de la transition à l'enseignement supérieur
}

\author{
Marie-Odile Magnan
}

Université de Montréal

\section{Résumé}

Plutôt que d'emprunter la lecture classique que propose généralement la sociologie de l'éducation - celle de l'égalité des chances et des mécanismes de reproduction sociale -, cet article analyse la construction identitaire linguistique des jeunes adultes au fil de leurs parcours scolaires et de mobilité. Les résultats d'une recherche qualitative $(\mathrm{N}=33)$ permettent de constater que la transition à l'enseignement supérieur, de par les différents rapports d'altérité qu'elle engendre, module la construction identitaire des jeunes issus d'un milieu scolaire linguistique minoritaire au Canada. C'est par une approche microsociologique et constructiviste que nous croisons les parcours objectifs (transitions scolaires, de mobilité) et subjectifs (construction identitaire linguistique) des jeunes en analysant leur récit de vie de manière diachronique. Bien que certains chercheurs aient évoqué la thèse du déclin de l'institution scolaire (Dubet, 2002), l'analyse montre que l'expérience sociale de l'école laisse une empreinte sur les parcours de vie.

\begin{abstract}
Rather than using the classical view generally embraced by the field of sociology of education - that is equity in educational chances and mechanisms of social reproduction -, this article analyses the construction of linguistic identity among young adults during their school and mobility pathways. The
\end{abstract}


results obtained from this qualitative research $(\mathrm{N}=33)$ demonstrate that the transition to higher education influences the construction of linguistic identity of young adults who previously attended a minority linguistic school in Canada - and this is mainly due to the new relationships to otherness that the transition to higher education involves. Using a micro sociological and constructivist approach, the article draws a parallel between the objective pathways (school and mobility transitions) and the subjective pathways (construction of linguistic identity) of young adults by analysing their life story with a diachronic approach. Although some researchers have evoked the decline of the school institution (Dubet, 2002), this article reveals that the social experience of school leaves a mark on life pathways.

Dans une perspective où l'institution scolaire est conçue comme un lieu de formation des identités collectives modernes (Thériault, 2002), il est pertinent de s’interroger sur le rôle des expériences scolaires formelles et informelles dans la (re)production des sentiments d'appartenance. Plutôt que d'emprunter la lecture classique que propose généralement la sociologie del'éducation - celle de l'égalité des chances et des mécanismes de reproduction sociale (Farmer et Heller, 2008) -, nous proposons dans cet article d'analyser la construction identitaire linguistique des jeunes au fil de leurs parcours scolaires et de leurs parcours de mobilité. Les résultats de recherche qui sont présentés dans cet article permettent de constater que la transition à l'enseignement supérieur, de par les différents rapports d'altérité qu'elle engendre, module la construction identitaire des jeunes issus d'un milieu scolaire linguistique minoritaire au Canada. Une étude qualitative des parcours identitaires de 33 jeunes universitaires canadiens ayant fréquenté l'école de langue anglaise de la ville de Québec révèle que les transitions à l'enseignement supérieur et la mobilité qui l'accompagne ont un impact sur la transformation des identités linguistiques. C'est par une approche microsociologique et constructiviste que nous croiserons les parcours objectifs (transitions scolaires, de mobilité) et subjectifs (construction identitaire linguistique) des jeunes en analysant leur récit de vie de manière diachronique (Bertaux, 2005). Bien que les recherches récentes sur les jeunes de minorités linguistiques privilégient des approches reconnaissant le caractère dynamique de la construction identitaire en tant que processus à la fois social et psychologique à l'adolescence (Dallaire, 2008; Landry, Deveau et Allard, 2006; Gérin-Lajoie, 2011; Pilote, 2007), les méthodologies les plus souvent empruntées permettent difficilement d'en analyser l'évolution dans le temps, au fil de la transition à l'enseignement supérieur. Notre article constitue ainsi une contribution novatrice à ce champ d'études par le biais d'une analyse rétrospective des parcours identitaires d'étudiants universitaires issus de l'école anglaise de Québec.

Dans le cadre du présent article, nous proposons donc une exploration des systèmes scolaires, de l'intérieur, en étudiant principalement les interprétations et parcours des acteurs individuels - en nous penchant sur une analyse de l'expérience sociale des institutions scolaires au fil du temps. Bien que certains auteurs aient évoqué la thèse du déclin de l'institution scolaire (Dubet, 2002), l'analyse que nous présentons montre que l'expérience sociale de l'école n'est pas sans effet sur les parcours de vie des jeunes adultes. 
Avant d'entrer au cœur de nos résultats, nous présentons le contexte éducatif dans lequel se situent les jeunes que nous avons interrogés. Puis, nous faisons état de la perspective théorique privilégiée, du cadre méthodologique et des résultats de recherche.

\section{Contexte éducatif}

En 1969, la Loi sur les langues officielles est adoptée par le Parlement canadien. Cette législation accorde, pour la première fois, une reconnaissance de l'anglais et du français comme langues officielles du Canada. C'est toutefois en 1982 que la Charte canadienne des droits et libertés vient consacrer officiellement l'importance de l'éducation de langue officielle minoritaire au Canada (Martel, 2001). En vertu des droits éducatifs inscrits à la Charte canadienne des droits et libertés, l'école a la responsabilité constitutionnelle d'assurer la reproduction linguistique et culturelle des communautés de langue officielle en situation minoritaire. En particulier, l'article 23 de cette Charte reconnait le droit à l'instruction dans la langue de la minorité et vise : 1) la transmission des valeurs linguistiques et culturelles (dans un établissement de la minorité), ainsi que 2) la réduction des pouvoirs de domination du groupe majoritaire (droit exclusif de gestion). Ces droits s'inscrivent en continuité avec les luttes historiques menées par la minorité francophone hors Québec, une minorité qui a fait de l'éducation son principal cheval de bataille pour assurer le maintien et le développement de ses communautés. Même si sur le plan canadien les anglophones du Québec jouissent du même cadre juridique que celui des francophones hors Québec, il reste que la minorité anglophone fut davantage marquée par le contexte politique et juridique québécois.

Depuis la Révolution tranquille, plusieurs changements au Québec ont marqué l'école de langue anglaise - des changements ayant suivi " l'évolution des rapports entre les communautés linguistiques » (Pilote et Bolduc 2007, p. 2). Dès les années 1960, la langue (plutôt queles questions d'ordre religieux ou économique) devient le principal objet delitige entre les anglophones et les francophones du Québec; la crise de Saint-Léonard marque notamment la fin d'une certaine harmonie sociale qui prévalait jusqu'alors (Stevenson, 1999) tandis qu'un conflit éclate entre ceux qui désirent intégrer les immigrants au réseau scolaire francophone et les italophones, qui réclament l'enseignement en anglais en tant que moyen d'ascension sociale.

Dans la foulée de ces conflits, la Loi 101 voit le jour en 1977. Cette loi proclame le français comme l'unique langue officielle du Québec et l'unique langue devant être visible sur les formulaires gouvernementaux, les affiches, les menus des restaurants, etc. Elle décrète, entre autres, le français comme langue de l'enseignement au Québec - les écoles privées non-subventionnées, les collèges et les universités n'y étant cependant pas assujettis. L'article 73 de la Charte québécoise de la langue française, ayant pour objectif d'intégrer les nouveaux arrivants à l'école de langue française, n'accorde l'accès aux écoles de langue anglaise qu'à la minorité anglophone ayant déjà fréquentéle système d'éducation anglophone par le passé. Les ayants droit à l'école publique de langue anglaise (primaire et secondaire) doivent ainsi répondre au critère suivant : avoir un parent ayant reçu la majorité de son enseignement primaire en anglais au Canada ${ }^{1}$.

À la suite de ces mutations, à quoi ressemble le système scolaire anglophone? Concernant le système d'enseignement public, le réseau anglophone compte 177 écoles primaires, 69écoles secondaires, 9 établissements d'enseignement collégial et 3 universités. 
Aux niveaux primaire et secondaire, les effectifs des institutions scolaires anglophones totalisent $10 \%$ de l'ensemble de la population des écoles primaires et secondaires du Québec (Lamarre, 2005).

Après l'entrée en vigueur de la Loi 101, le nombre d'inscriptions dans le réseau anglophone diminue. Les effectifs scolaires des écoles de langue anglaise augmentent cependant de nouveau dans les années 1990. Des changements juridiques expliquent entre autres cette récente croissance : des clauses de la Loi 101 sont amendées en ce qui a trait aux élèves ayant des difficultés d'apprentissage sévères et aux étudiants séjournant temporairement au Québec. Puis, les mariages entre francophones et anglophones ainsi qu'entre allophones et anglophones contribuent à l'augmentation du nombre d'ayants droit. Ces variations au sein de la population scolaire ont comme conséquence de réduire la proportion d'anglophones dans les écoles de langue anglaise (principalement à l'extérieur de Montréal). Cette forte présence francophone dans les écoles de langue anglaise à l'extérieur de la région de Montréal a des implications sur la mission du réseau scolaire anglophone alors que l'on observe un brouillage des frontières linguistiques à l'intérieur même des murs de l'école.

Aujourd'hui, l'éducation de langue anglaise constitue l'un des principaux vecteurs pouvant permettre la préservation ou la création d'un sentiment d'appartenance au groupe anglophone. À l'extérieur de Montréal, l'école forme le lieu principal de rencontre quotidien de la minorité. Or, peu d'études ont porté jusqu'à présent sur le rôle de l'école de langue anglaise dans la production d'un sentiment d'appartenance. Tout récemment, quelques chercheurs ont néanmoins abordé la question de la construction identitaire des jeunes adolescents en milieu scolaire de langue anglaise au Québec (Gérin-Lajoie, 2011; Vieux-Fort et Pilote, 2010; Pilote et al., 2010).

Nous proposons donc ici de suivre ces élèves qui ont fréquentél'école de langue anglaise au secondaire à travers leurs parcours à l'enseignement supérieur ainsi qu'à travers leurs parcours de mobilités (ou d'immobilité). En effet, au niveau secondaire le jeune se situe à l'adolescence, une étape de la vie où tout est incertain, où tout se transforme de façon quotidienne, où tout est en devenir : « Il s'agit [...] d'un moment où l'identité est non seulement inachevée (ou non cristallisée), mais aussi, dans certains cas, problématique et profondément remise en question » (Pilote, 2007, p. 235). Durant la période des transitions menant vers la vie adulte, une période charnière de socialisation de la jeunesse, «l'identité se construirait, par approximations successives, au gré des diverses expériences sociales qui suivent la décohabitation familiale » (Galland, 1990, p. 529). Si, dans le cadre du présent article, nous considérons que le lien entre les transitions menant vers la vie adulte et les transformations identitaires n'est pas unidirectionnel (Molgat et Vézina, 2008), nous nous proposons néanmoins de mettre en relief l'articulation entre la construction identitaire d'une part, et les transitions à l'enseignement supérieur et de mobilité d'autre part. À l'instar de Molgat (2007), nous percevons la construction identitaire du jeune adulte comme un phénomène relevant à la fois de conditions structurelles et de réactions individuelles.

\section{Un angle microsociologique : la construction identitaire linguistique au fil du parcours à l'enseignement supérieur}

Notre cadre théorique s'inscrit dans une approche constructiviste - une approche où l'on reconnaît un rapport dialectique entre le social et l'individuel, et où l'on admet 
que le social produit les individus qui, en retour, transforment et produisent le social (Corcuff, 1995). Cette étude vise plus spécifiquement à mieux comprendre les processus par lesquels les jeunes construisent leur identité linguistique au fil de leur parcours de vie, à partir de socialisations plurielles provenant de l'interaction avec leurs pairs, divers intervenants scolaires, des membres de leur famille, etc. (Darmon, 2006). Nous concevons la construction identitaire linguistique comme une expérience sociale inachevée, résultant du « travail » continu de l'acteur construisant son unité à l'aide de différents éléments de sa vie (Dubet, 1994). Le jeune est donc considéré ici comme un acteur social participant à sa construction identitaire linguistique à l'intérieur des contraintes de la structure sociale et, plus particulièrement, des institutions scolaires.

Nous envisageons ainsi la construction identitaire linguistique comme un processus biographique d'incorporation des appartenances linguistiques issues de l'ensemble des systèmes d'action traversés par l'individu au cours de son existence (Dubar, 2010). L'école est toutefois considérée ici comme un agent principal de transmission identitaire et langagière (Landry et al., 2010), l'institution scolaire étant un " espace de mise en contact et d'articulation des autres formes de socialisation » (Darmon, 2006, p. 62). Nous proposons donc de faire une sociologie de l'école (Duru-Bellat et VanZanten, 2006) où les systèmes scolaires sont explorés de l'intérieur, sous l'angle de l'interprétation subjective des jeunes. Quelle est l'identité linguistique que les jeunes développent en lien avec leur expérience scolaire, à travers les interactions quotidiennes avec les pairs et les divers acteurs scolaires? Précisons que dans notre étude, l’identité linguistique ne renvoie pas à la langue maternelle ou au sentiment de compétence langagière, mais bien au sens d'appartenance, subjectif à un ou à plusieurs groupes linguistiques.

L'identité linguistique est alors définie comme un processus complexe et dynamique qui s'exerce tout au long des interactions de l'existence et « qui lie étroitement la relation à soi et la relation à autrui » (Lipiansky, 1998, p. 21) dans un processus de rapprochement et de différenciation face à l'Autre. L'identité socialement construite est donc le produit d'une négociation constante entre des attributions linguistiques provenant d'autrui et l'identification subjective de l'individu (Strauss, 1997; Dubar, 1996). L'identitélinguistique se construit et s'articule à l'aide d'appartenances linguistiques qui, soit lui servent de contraintes, soit lui servent de ressources créatrices menant éventuellement vers des appartenances multiples.

Selon notre approche, l'identité linguistique se développe de façon réflexive en regard du parcours de vie de l'individu ainsi qu'en regard du cadre spatiotemporel qui l'entoure. Pour mieux tenir compte de l'histoire personnelle des individus, nous retenons la notion de schémas de soi (traduction du terme self-schemas) qui, selon Kaufmann (2004) : « résultent en effet de la trajectoire sociale, de l'histoire de la personne. Ils ne sont au fond que le reflet d'expériences de confrontations avec divers contextes enregistrés sous forme de cadre de détermination des actions futures » (p. 77). Dans la modernité, les individus sont guidés par leur propre parcours biographique qu'ils ont intériorisé, dans un dialogue constant entre leur encodage social passé et présent. Ce sont les individus eux-mêmes, de par leur expérience passée, qui édifient les balises limitant leur action.

Suivant cette approche constructiviste de l'identité linguistique, nous avons considéré la transition vers l'enseignement supérieur et la transition de mobilité spatiale, qui peut l'accompagner, comme des étapes charnières de la construction identitaire. La socialisation 
qui caractérise ces expériences éducatives et territoriales peut en effet amener les jeunes à se voir sous un nouveau jour - la découverte de nouvelles alternatives pouvant contribuer au développement d'appartenances (ou de non-appartenances) à de nouveaux groupes linguistiques. De plus, la transition associée à l'enseignement supérieur se caractérise par une période où les dilemmes et les négociations identitaires sont importants (PerretClermont et Zittoun, 2002). Nous postulons que ces étapes objectives concourent de près à la formation, à la transformation, voire à la consolidation de l'identité et des appartenances linguistiques. L'enseignement supérieur et la mobilité peuvent donc amener le jeune adulte à réviser ses appartenances. En effet, les nouveaux rapports d'altérité vécus par les jeunes au cours de leurs études postsecondaires peuvent conduire à une prise de conscience plus aiguë des frontières entre les divers groupes de référence linguistiques. Ce faisant, les jeunes sont confrontés à des dilemmes et sont soumis à la négociation de leur identité linguistique au cours des nouvelles interactions sociales qui jalonnent leur parcours à l'enseignement supérieur.

Chez les jeunes issus d'une scolarisation en contexte linguistique minoritaire, les expériences sociales liées à la transition à l'enseignement supérieur peuvent susciter un questionnement sur le plan de l'appartenance au groupe linguistique d'origine ainsi qu'à l'appartenance à de nouveaux groupes linguistiques. Au Canada, ce questionnement concerne l'appartenance aux groupes francophones et anglophones, bien qu'il ne s'y limite pas. Alors que plusieurs études ont porté jusqu'à présent sur l'identité des minorités francophones au Canada à l'école primaire et secondaire (par exemple, voir Heller, 2003; Gérin-Lajoie, 2003 et 2004; Pilote, 2006 et 2007; Landry, Deveau et Allard, 2006; Dallaire, 2008; Pilote, Magnan et Vieux-Fort, 2010), il reste que la construction de l'identité linguistique lors des études universitaires a fait l'objet de très peu de recherches. L'étude de Lamoureux (2005) sur le parcours universitaire d'étudiants provenant de minorités francophones au Canada fait exception. Elle révèle que l’identité linguistique évolue après les études secondaires. Par une étude qualitative del'identité et del'affirmation linguistique d'étudiants du Sud-Ouest ontarien transitant de l'école secondaire de langue française vers l'université, elle montre qu' « au fil de l'année [universitaire] et de leurs contacts avec toutes sortes de personnes dans des contextes différents, les étudiants voient de moins en moins les frontières linguistiques qui existent entre les diverses communautés auxquelles ils appartiennent » (Lamoureux, 2005, p. 119).

Nous concevons ainsi la construction identitaire linguistique des jeunes scolarisés en anglais au Québec à l'adolescence comme un processus en mouvance qui doit être situé dans son contexte temporel, voire dans les schémas de soi (parcours biographique) qui animent les acteurs sociaux. C'est en partant de cette approche constructiviste, qui replace la construction identitaire linguistique dans son parcours biographique, que nous avons procédé à l'analyse diachronique des parcours où nous avons mis en parallèle 1) les transitions de vie objectives générant des mouvements concrets dans le temps et l'espace (transitions scolaires, de mobilité, etc.) et 2) les transitions identitaires sur le plan linguistique (Pilote et Magnan, 2012).

\section{Méthodologie}

Des entrevues semi-dirigées ont été menées auprès de jeunes universitaires scolarisés à l'école secondaire de langue anglaise de Québec. Le terrain de recherche s'est déroulé 
entre les mois d'avril 2008 et de mars 2009. Au total, le corpus qualitatif comprend 33 entretiens individuels semi-dirigés. Et environ les deux tiers des répondants proviennent d'un foyer bilingue où un parent parle l'anglais et l'autre, le français. La majorité des jeunes interrogés déclarent avoir une bonne connaissance du français. Nous avons recueilli des témoignages répondant à plusieurs critères : jeunes qui ont fait des études universitaires en français/études universitaires en anglais/études universitaires en français et en anglais; immobilité/mobilité au sein du Québec et au sein du Canada. Ainsi, au moment de mener l'entretien, le tiers des jeunes interrogés étudiaient dans une université francophone du Québec, le tiers dans une université anglophone du Québec et le tiers dans une université anglophone ou bilingue située dans une autre province canadienne que le Québec. Au total, les deux tiers de nos répondants sont de sexe féminin. Vingt-et-un répondants sont âgés de 20 à 22 ans, alors que douze d'entre eux sont âgés de 23 à 27 ans. De plus, environ les deux tiers proviennent d'un foyer où au moins l'un des parents détient un diplôme universitaire ${ }^{2}$.

La population à l'étude est donc celle des jeunes universitaires (de 20 à 29 ans) qui ont fait leurs études secondaires en anglais dans le contexte local de la ville de Québec. Plusieurs raisons justifient cette étude de cas. D'une part, l'ensemble de la minorité anglophone de la capitale nationale se distingue fortement de celle de Montréal. Elle se caractérise par un niveau d'identification plus élevé au groupe francophone (Corbeil et al., 2007) et par un taux plus élevé de bilinguisme (89,4\%). Environ $40 \%$ des anglophones de Québec sont issus d'un couple exogame (c'est-à-dire d'un couple mixte sur le plan linguistique) (Jedwab, 2002). D'autre part, ces mélanges linguistiques ont un impact sur la population estudiantine de la région alors que les pourcentages de francophones et d'anglophones dans les écoles de langue anglaise sont respectivement $63 \%$ et $37 \%$ (Jedwab, 2002). Ainsi, la minorité anglophone de Québec, évoluant dans un contexte majoritairement francophone, traverse les frontières linguistiques de façon quotidienne - que ce soit dans les lieux publics, à l'école ou à la maison. Cette situation est susceptible selon nous de donner lieu à des bricolages identitaires riches et complexes en terme de construction identitaire à l'âge adulte.

\section{Analyse des parcours identitaires linguistiques au fil des transitions scolaires}

Nous avons effectué une analyse rétrospective des parcours scolaires et identitaires racontés par les jeunes de notre corpus. Les thèmes abordés en entrevue étaient les suivants : parcours de vie durant l'enfance et l'adolescence, parcours de mobilités ou de non-mobilité lors de la transition à l'âge du jeune adulte, parcours à l'enseignement supérieur et parcours identitaires linguistiques. Afin de réaliser une analyse diachronique des mises en récit, nous nous sommes largement inspirées de la méthode d'analyse élaborée par Bertaux (2005). Les récits de vie permettent en effet de dégager une dimension diachronique des parcours, qui mène vers la découverte de logiques d'actions situées à des moments précis du parcours de vie. La démarche rétrospective intrinsèque aux récits de vie permet d'analyser le discours réflexif des individus (leur récit narratif). En ce sens, cette dimension diachronique des récits de vie nous permet ici de mieux comprendre le «processus » de construction identitaire linguistique. Il faut cependant mentionner qu'il s'agit là de ce qu'en disent les répondants au présent, donc a posteriori - ce qui suggère un effet de la narration identitaire (Kaufmann, 1996), un biais méthodologique dont nous 
devons tenir compte lors de l'analyse des données. Plutôt que de se laisser prendre à la reconstruction d'une certaine " idéologie biographique », voire à cette tendance où " chacun tend à se représenter le cours de son existence comme présentant une forte cohérence » (Bertaux, 2005, p. 38), nous avons plutôt procédé à la déconstruction de leurs parcours en menant une analyse diachronique minutieuse.

Une identité « anglophone » à l'adolescence : l'expérience sociale de l'école de langue anglaise de Québec. Nous pouvons d'abord dégager le constat suivant pour l'ensemble de notre corpus : l'expérience de l'école secondaire de langue anglaise de Québec génère à l'adolescence une configuration de relations sociales menant vers une identité linguistique « anglophone ». Le cadre institutionnel et structurel que constitue l'école de langue anglaise contribue à générer des interactions communes entre, d'une part, les élèves de cette école linguistique évoluant en contexte minoritaire et, d'autre part, les élèves de l'école de langue française et la majorité francophone. À l'instar de VieuxFort et Pilote (2010), notre étude montre que l'unique fait de fréquenter l'école de langue anglaise constitue le marqueur d'une frontière identitaire, car dans le regard de l'autre francophone majoritaire « aller à l'école de langue anglaise signifie être un anglophone » : "I think in Anglophone schools, you are already placed against everyone that's French » (Julia ${ }^{3}$, Université Laval). Le récit que Julia fait de son expérience de deux ans dans une école secondaire francophone permet de comprendre davantage l'importance de l'école dans le jeu des identifications et des assignations identitaires linguistiques :

[By going to French school] I felt like I was more part of Quebec because I was in French school and I was like « oh I am now like every other kid ». You know, before, you feel so different because you are a minority and when you are in an English school «oh, there is the minority ». [At French school] I felt like a normal kid [...] I got more normal (laughs).

Les interactions entre les élèves des écoles de langue anglaise et de langue française engendrent des catégorisations linguistiques et des tensions sur le plan du rapport d'altérité entre les membres et non-membres des deux groupes : " there were fun things like going to Village des sports. When you'll have different schools like the French schools and English schools. And they'd hear the English school speaking English and you know 'les maudits anglais' and all that »(Cindy, Université Bishop). Andrea (Université Concordia) raconte comment ses amis francophones perçoivent les élèves fréquentant les écoles de langue anglaise : " they'd be like “oui, mais tu sais, le monde à Quebec High School puis à St-Patrick sont bizarres là les Anglais” ». Anthony (Université d'Ottawa) raconte comment, dans son enfance, les groupes des écoles de langue anglaise et française se définissaient l'un contre l'autre en s'affrontant dans la cour d'école :

When I was a child, up to grade 4, it was English and French together. On the same school yard, which was particularly unique because it'd be French against English for everything... it was still the old you know English versus French and the snowball fights... everything would be on these linguistic lines.

Parfois, les affrontements entre les écoles tournent à la bataille : 
Nous, quand on était à l'école, ce qui était francophone si on veut, c'est les polyvalentes d'autour. Quand ils venaient, c'était tout le temps pour nous faire des graffitis sur les murs ou pour se battre, puis tout plein d'affaires là. C'était jamais super positif (Annie, Université Laval).

L'expérience de l'école de langue anglaise génère chez 18 de nos répondants - durant la période de l'adolescence - une vision dichotomique des communautés « francophone québécoise » et " anglophone canadienne », une vision par laquelle il leur semble alors impossible d'être à la fois " anglophones canadiens » et " francophones québécois » : « it's weird because...[to have a French identity] like it contradicts everything English about me. Because people think you are either English or French, you can't be both » (Maude, Université McGill).

Dans notre corpus, nous retrouvons ainsi une majorité d'étudiants dont le parcours et la construction identitaire furent marqués par le passage à l'école de langue anglaise. Dans le contexte de la ville de Québec, les interactions sociales avec les francophones contribuent à créer (chez les étudiants de l'école de langue anglaise) le sentiment de former un " groupe anglophone » distinct de celui des francophones fréquentant l'école de langue française. Même si certains étudiants sont transformés par de nouveaux rapports d'altérité lors de leurs parcours postsecondaires et de mobilité, il reste que l'expérience de l'école de langue anglaise dans le contexte local de Québec - une expérience forte au plan des rapports d'altérité - constitue un repère dans leur schéma de soi - un repère duquel ils se rapprochent ou se distancient lors de leur transition à l'enseignement supérieur.

Parcours identitaires linguistiques lors de la transition à l'enseignement supérieur. Après avoir fait l'expérience sociale de l'école de langue anglaise à Québec, les jeunes qui transitent à l'enseignement supérieur conservent-ils cette vision dichotomique des identités " francophone québécoise » et « anglophone canadienne »? Trois types de processus identitaires ont émergé de notre corpus qualitatif : 1) certains jeunes, durant leur parcours à l'enseignement supérieur, conservent une vision dichotomique des identités " francophone québécoise » et " anglophone canadienne » (8 cas); 2) certains jeunes adoptent une vision plurielle de leur identité linguistique tout au long de leur parcours, une vision par laquelle il est considéré possible d'être à la fois " francophone québécois » et " anglophone canadien ", deux identités qui ne seraient pas vues comme étant mutuellement exclusives ( 7 cas) et 3) certains jeunes passent de la vision dichotomique à la vision plurielle de leur identité linguistique tout au long de leur parcours (18 cas).

Ainsi, l'analyse de données révèle que les deux tiers des jeunes de notre corpus passent d'une vision dichotomique des identités linguistiques à une vision plurielle où les identités « francophone québécoise » et " anglophone canadienne » se combinent et ne sont pas mutuellement exclusives. Prenons l'exemple de Florence. Après avoir étudié à l'université dans une autre province canadienne (nous ne mentionnons pas l'université pour des raisons de confidentialité), Florence indique avoir fait un retour réflexif sur son identité. Alors qu'elle se définissait comme anglophone à l'adolescence, elle se décrit désormais comme suit :

Je suis les deux. Je suis Canadienne, je suis Québécoise. Je peux dire : «j’aime bien ça parler en français, mais Québec est dans le Canada. Je parle en anglais. Je fais partie des deux ». Puis c'est pas juste, je fais partie des deux. Dans ma tête, c'est une place. 
Elle raconte notamment comment le contenu de ses cours universitaires l'a amenée à faire ce retour réflexif sur son identité linguistique :

[...] puis, il y a des choses que j'ai appris sur le Québec [dans cette province canadienne]. Que j'ai trouvé ça super intéressant parce que j'avais un prof qui montrait toutes les parties francophones... mettons à Moncton, dans l'Ouest... y montrait le français pas mal au Canada, comment que c'était un peu partout. Puis là, le même prof il a donné un cours sur la littérature québécoise ou histoire québécoise...[...] j'ai plus appris des choses là-bas. Puis j'étais super intéressée. Là, je m’identifiais plus [comme francophone québécoise].

Florence, lors de son passage à l'université, s'oppose fortement à la vision dichotomique des communautés imaginées «francophone québécoise » et « anglophone canadienne » :

Dans un des cours en français, il y avait une fille... ça a adonné la seule journée que j'ai manquée, j'étais pas là... mais ils parlaient de moi parce qu'ils parlaient du Québec... elle était là " elle vous pensez qu'elle est comment? », «bien, elle est Canadienne, Québécoise ». Puis là, il y a une fille qui disait " non, elle est juste Québécoise. Elle parle en français... elle est pas Canadienne ». Bien là, moi ça m’a fâchée... je suis Canadienne, je suis pas juste Québécoise. Je suis fière d'être Québécoise, mais je suis fière d'être Canadienne aussi... je fais partie des deux... dans ma tête c'est une place. Québec est dans le Canada.

L'exemple d'Andrea, de l'Université Concordia, montre aussi comment s'effectue ce passage de la vision dichotomique des identités linguistiques à la vision plurielle. À Québec, où le français constitue la langue majoritaire, elle se sentait minoritaire par le fait notamment d'aller à l'école de langue anglaise : « the fact that I went to an English school, that really set me apart, that I really felt a little out of place ». Elle se définit principalement contre le regard des francophones qui lui accolent l'étiquette d'anglophone. Elle affirme que ce rapport d'altérité la pousse d'autant plus à se définir en tant qu'anglophone à l'adolescence : « Like I feel it just made me feel stronger about my roots and made me even more proud to be an Anglophone... I hated them and that made us close in even more and not want to integrate ».

Elle choisit par la suite de poursuivre ses études dans une université anglophone de Montréal : l’Université Concordia. Auparavant, elle se serait définie comme étant une anglophone provenant de la ville de Québec, alors que maintenant elle considère être devenue une jeune urbaine de langue anglaise : " when I moved here, you know, I embraced other cultures ». Elle sent qu'elle se reconstruit désormais selon ses nouvelles expériences et selon sa propre subjectivité, sans se faire imposer des étiquettes qui la définissent $a$ priori :

I don't feel there is one thing in particular that defines me. I don't think I've decided quite yet on what defines me. I am still taking it all... I am building my own identity with things that I feel a connection with, not just something I was told all my life. Now, it's me who decides who I am.

$\mathrm{Si}$, dans le contexte local de la ville de Québec, Andrea se sentait conditionnée à être une « anglophone de Québec » en raison de son univers familial et scolaire ainsi qu'en 
raison de l'étiquette linguistique que lui accolaient les francophones de Québec, sa mobilité montréalaise et son expérience de l'université l'ont menée vers une transformation de son identité individuelle. Si elle se voit elle-même comme étant en train de se redéfinir, cela peut s'expliquer en partie par la mobilité qu'elle a effectuée; car selon son récit, c'est lorsqu'elle a migré pour un milieu davantage cosmopolite qu'elle a commencé à se définir autrement - voire à se libérer des frontières entre les « anglophones canadiens » et les « francophones québécois ».

C'est ainsi que 18 jeunes que nous avons interrogés racontent faire ce passage de la vision dichotomique à la vision plurielle lorsqu'ils passent à l'âge adulte et lorsqu'ils vivent une transition à l'enseignement supérieur - une transition impliquant notamment une transition de mobilité.

Cette analyse souligne le rôle crucial des transitions scolaires et de mobilité dans les processus identitaires linguistiques vécus par les jeunes. Elle permet aussi de réaliser que plus les individus diminuent leurs interactions avec leur groupe d'appartenance linguistique, plus ils en viennent à s'identifier à de multiples groupes de référence, c'est-àdire à plusieurs groupes qui leur fournissent « [des] valeurs, [des] normes, [des] modèles d'attitude, d'opinion et de comportement » (Lipiansky, 1998, p. 149). Ainsi, c'est en interagissant avec d'autres " autruis ", à la suite de transitions scolaires ou de transitions de mobilité, qu'ils s'inscrivent davantage dans une vision plurielle des appartenances. Sans quitter pour autant leur groupe d'appartenance lié à la minorité de langue anglaise de Québec, l'éloignement social ou géographique contribue, chez les jeunes qui sont passés de la vision dichotomique à la vision plurielle, à un repositionnement identitaire menant la plupart du temps vers de multiples affiliations identitaires. Dès lors que la frontière qui sépare les groupes linguistiques n'est plus visible dans des lieux de contacts et dans des situations d'interactions entre des groupes de jeunes francophones et des groupes de jeunes anglophones, les groupes d'appartenance deviennent soudainement combinables et non plus mutuellement exclusifs.

Alors qu'à l'adolescence l'identité linguistique de la majorité des jeunes de notre corpus semble être prédéterminée par la structure interactionnelle que leur propose l'expérience de l'école de langue anglaise de Québec, la période des études universitaires semble plutôt être associée à un bricolage identitaire où la subjectivité de l'acteur social entre en scène pour contester cette vision dichotomique des identités « francophone québécoise » et « anglophone canadienne». Ces transformations identitaires sont-elles le résultat du passage à l'âge adulte, des transitions ou de l'éloignement social ou géographique? Est-ce le fait de quitter ou de se distancer des interactions liées à l'expérience de l'école de langue anglaise qui contribuerait plutôt à ces repositionnements? Car, en l'absence de contacts avec les autres « anglophones » de Québec, l'identité de groupe semble s'effriter.

Les données présentées dans cet article permettent de penser qu'il y a à la fois un effet de période de vie et un effet de transition. D'une part, l'adolescence constitue un moment où le jeune est fortement influencé par le regard de ses pairs; ici, le jeune fréquentant l'école de langue anglaise se révèle marqué par l'assignation d'une étiquette « anglophone » par son homologue francophone ainsi que par la vision dichotomique qui semble exister sur le plan des identités linguistiques. Selon la mise en récit d'une majorité de nos répondants, il semble que l'adolescence soit une période où l'on s'approprie cette vision sans la remettre en question. L'adolescence est aussi un moment où le jeune fréquentant l'école de langue 
anglaise est plus susceptible d'être en groupe (et non pas comme un individu) face à des francophones qui sont eux aussi en groupe (dans des lieux de contacts comme l'autobus, la rue, les tournois sportifs, la cour d'école, etc.).

Les identités dichotomiques sont donc plus vives et plus susceptibles d'être expérimentées pendantl'enfance et l'adolescence, c'est-à-dire au moment où l'on fréquente l'école. L'âge où le jeune adulte effectue une transition vers l'enseignement supérieur peut être associé à une plus grande réflexivité, c'est-à-dire à une étape de la vie où le jeune voit sous un nouveau jour son appartenance, révise la vie sociale à la lumière de nouvelles informations et situations (Giddens, 1991), de nouvelles alternatives pouvant contribuer au développement d'appartenances (ou de non-appartenances) à de nouveaux groupes.

D'autre part, les transitions scolaires et de mobilité semblent également cruciales dans la transformation des identités linguistiques des jeunes interrogés et dans l'agencement entre différents groupes d'appartenances et de références. Les données montrent que la dichotomie entre "francophone québécois » et « anglophone canadien » se met à s'effriter et à perdre son sens dans les histoires des répondants dès qu'ils découvrent un cadre scolaire ou territorial qui sous-tend d'autres altérités (des individus provenant de l'étranger, des anglophones d'autres provinces canadiennes, des anglophones venus de l'extérieur du Canada, etc.). Ils sortent ainsi de la dichotomie dès qu'ils passent dans un contexte où la langue n'est plus utilisée comme l'unique marqueur identitaire.

L'essentiel de l'analyse présentée ici révèle que les logiques identitaires supposant la dichotomie et le sentiment d'appartenance au groupe anglophone n'ont de sens que dans un milieu construit autour d'une prétendue homogénéité linguistique (celui de la ville de Québec) et où seuls les anglophones semblent s'opposer aux francophones majoritaires. Mais surtout, les résultats montrent que le pluralisme d'un contexte dans lequel il n'y a plus uniquement une opposition entre les « anglophones » et les « francophones » contribue fortement à dissoudre la dichotomie. La représentation dichotomique des identités linguistiques qui semble exister à Québec nous semble être singulièrement liée au territoire particulier de la ville de Québec et à la période que nous étudions - elle est donc située dans un temps sociohistorique et territorial bien déterminé.

Dans une majorité de mises en récit du corpus, on peut constater qu'au moment où l'Autre de la majorité francophone cesse d'être uniquement l'anglophone (qu'il devient aussi par exemple l'Autre immigrant dans un contexte canadien urbain multiethnique), il ne va pas de soi que la seule existence des institutions anglophones puisse fonder le même genre d'interactions dichotomisantes - et ainsi le même sentiment d'appartenance au groupe linguistique « anglophone ». Les répondants sortent de la dichotomie et se mettent à envisager des appartenances combinables ou des identités de « citoyens du monde », principalement quand ils ont l'impression de découvrir la diversité, ce qui se produit quand ils peuvent se projeter dans un espace autre que celui de la ville de Québec ou celui des écoles de langue anglaise de Québec.

Prenons l'exemple de Nathalie, de l'Université Concordia, qui se définissait comme anglophone lors de son adolescence passée dans la ville de Québec. Depuis qu'elle a migré vers Montréal, elle se définit désormais en fonction d'un amalgame de trois appartenances : le pays d'origine de sa mère, le Canada anglais (traduction de « English Canada ») et le Canada français (traduction de «French Canadian ») : «I don't have a clear identity anymore... I am kind of a mixture of all three ». Son expérience montréalaise semble avoir 
contribué à ce repositionnement identitaire : «there is (sic) so many different cultures, it would be easy to build my own identity without having... without feeling so different, because everybody is different ».

C'est ainsi que dans un milieu où les appartenances se multiplient, et ne sont plus exclusives, le jeune « élargi[t] sa marge de jeu personnelle » (Kaufmann, 2004, p. 148). Il n'est plus piégé ou contraint par une identité linguistique unique. L'analyse des données suggère ainsi un écart entre les appartenances linguistiques disponibles dans un milieu comme Québec et les appartenances disponibles dans un milieu cosmopolite comme Montréal. L'effet de période de vie ainsi que les transitions scolaires et de mobilités semblent donc jouer un rôle important dans la transformation des identités des jeunes universitaires interrogés et dans l'agencement entre différents groupes d'appartenance.

Comment alors expliquer pourquoi certains individus de notre corpus (8 cas) conservent une vision dichotomique des identités " francophone québécoise » et " anglophone canadienne »? Au terme de l'analyse, nous pouvons constater à cet égard que ces jeunes sont restés en interaction constante avec leur groupe d'appartenance lié à l'école de langue anglaise de Québec, malgré les transitions à l'enseignement supérieur et les transitions de mobilité qu'ils ont effectuées.

Si David et Karen, par exemple, ont quitté Québec pour poursuivre des études universitaires en anglais, ils reviennent tout de même - les fins de semaine et pendant l'été - dans leur ville natale; leur nouveau lieu de résidence n'apparaît donc qu'être un lieu de passage lié aux études universitaires. Ainsi, leur entourage immédiat (famille, amis, etc.) ne semble pas avoir changé. Ces jeunes s'investissent encore principalement dans les mêmes interactions ayant marqué leur adolescence. Les nouveaux " autruis » rencontrés dans de nouveaux territoires ou dans de nouvelles institutions scolaires n'ont pas suscité de retour réflexif sur leur(s) identité(s) linguistique(s); ces répondants sont restés campés dans une vision dichotomique des identités « francophone québécoise » et « anglophone canadienne ». L'extrait d'entrevue suivant illustre bien la conservation de la vision dichotomique des identités linguistiques, même après une migration pour études universitaires :

If I say I am from Quebec, people « oh you are Québécois, you're French ». And I am like « no, I am not French, I am English»... I say... I am Anglophone. I don’t say I am Québécois... not that I don't want to be associated with Quebec. But if I say « I am Québécois », people assume that I am French. But I am not francophone, I am Anglophone (Karen, Université McGill).

Même vision dichotomique des identités linguistiques chez David, qui a effectué une migration vers l'Université Bishop, qui est bilingue et dont les amis sont majoritairement francophones :

It's such a part of your everyday life when you speak English, that there is nothing you can do about it. You just get used to it. It's like, you know, if somebody keeps telling you something you either stand up and fight or you accept it and you move on. I have accepted that I am a minority, that will not change... there is nothing anybody can do about it. 
Comment expliquer que certains individus plutôt que d'autres s'inscrivent dans ce processus identitaire statique - un processus dans lequel peu de changements s'observent sur le plan de la construction identitaire linguistique? D'abord, on constate que ces répondants proviennent soit d'un foyer unilingue anglophone, soit d'un foyer où l'un des parents (ou un grand-parent) a fortement influencé la construction d'une appartenance linguistique anglophone. Ainsi, sur les cinq répondants de notre corpus provenant d'un foyer unilingue anglophone, trois d'entre eux se retrouvent dans ce processus. La mise en récit du parcours de ces répondants met en relief que le fait d'avoir été élevés uniquement en anglais ne leur permet pas, a posteriori, de se reconnaître une identité « francophone québécoise ». Quant aux autres répondants qui s’inscrivent dans ce profil, ils racontent avoir été fortement influencés par un parent, ou un grand-parent, voulant leur léguer une appartenance anglophone. On observe ainsi chez ces répondants un rapport de continuité avec l'expérience familiale et scolaire vécue durant l'enfance et l'adolescence.

Enfin, en ce qui concerne le processus incluant des répondants qui adoptent une vision plurielle des identités « francophone québécoise » et « anglophone canadienne » depuis l'enfance (7 cas), on peut l'expliquer principalement par le fait que ces répondants proviennent majoritairement de foyers mixtes où les parents (dont l'un est francophone et l'autre anglophone) semblent léguer de manière égalitaire leurs langues et leurs cultures : « je suis dans un groupe de personnes on peut pas... je peux pas me traiter de francophone ou d'anglophone, je suis vraiment les deux » (Thomas, Université Laval). Il est à noter, toutefois, que la plupart des jeunes interrogés provenant de foyers bilingues (dont un parent est francophone et l'autre est anglophone) ont expérimenté une socialisation familiale principalement anglophone.

\section{Conclusion}

Pour une majorité des jeunes de notre corpus, on observe une multiplication des appartenances au cours des transitions à l'enseignement supérieur et des transitions de mobilités - une multiplication de l'ordre du cumul et de la combinaison, et non pas del'ordre de l'exclusion mutuelle. Ces jeunes considèrent qu'ils peuvent être à la fois francophones, anglophones, Québécois, Canadiens, etc. Dès lors que les jeunes ne sont plus contraints par l'univers interactionnel qu'est l'école de langue anglaise, ils sont amenés à juxtaposer leurs identités multiples. Dès lors qu'ils croient découvrir la diversité, les répondants sortent de la dichotomie et se mettent à envisager des appartenances combinables - ce qui se produit quand ils se projettent dans un espace autre que celui de la ville de Québec. Il semble en effet que ce soit la découverte de la diversité et l'expérience d'autres rapports d'altérité dans un nouveau cadre universitaire et territorial qui permettent aux jeunes ayant fréquenté l'école de langue anglaise de Québec de 1) se percevoir différemment, c'est-à-dire plutôt comme une minorité parmi plusieurs dans un monde où chacun serait différent et où il n’y aurait plus de majorité; 2) se définir selon des identités multiples. Ce remaniement de soi et ce repositionnement dans l'espace social (Perret-Clermont et Zittoun, 2002) auront inévitablement une influence sur la vie future de ces jeunes (Wiborg, 2001).

Ces résultats, replacés dans les débats autour de la construction identitaire du jeune adulte (Molgat, 2007; Arnett, 2007), permettent de penser que l'identité linguistique à cette étape de la vie est à la fois le résultat d'aspects structuraux (ex. : transitions scolaires et de mobilité impliquant un changement de milieu de vie qui induit une perception différente des groupes linguistiques) et le résultat d'aspects individuels liés à l'âge et à la 
façon subjective dont le jeune manipule ces transformations au profit de la construction de son identité linguistique. Les résultats mettent également en lumière la manière dont s'articulent, chez nos répondants, la construction identitaire et les transitions scolaires et de mobilité. En ce sens, il apparaît que les jeunes sont bel et bien marqués par l'expérience sociale qu'ils vivent au sein des institutions scolaires qui jalonnent leur parcours de vie. Il serait d'ailleurs pertinent d'examiner, dans le cadre de futures recherches, à quel point nos conclusions peuvent aussi s'appliquer à d'autres groupes que celui des étudiants universitaires issus de l'école de langue anglaise.

Ultimement, au regard des résultats de la recherche dont il est question ici, on peut constater l'importance du rôle de l'éducation en contexte minoritaire dans la formation des identités linguistiques et dans le parcours des jeunes à l'enseignement supérieur. Au niveau provincial, cette étude met également en lumière le rôle crucial de l'école anglaise dans la (re)production des frontières linguistiques entre francophones et anglophones. Les résultats de cette recherche interpellent en outre le travail des conseillers et des conseillères en orientation. En effet, nos résultats soulignent l'importance, pour ces professionnels œuvrant dans le réseau scolaire anglophone, de prendre en compte les dimensions linguistique et identitaire des parcours scolaires antérieurs de ces jeunes, afin de pouvoir mieux les guider en leur présentant l'ensemble des choix qui s'offrent à eux, dont celui des études en français. Cette question constitue un enjeu social majeur pour la société québécoise qui souhaite préserver la vitalité de la langue française à l'échelle provinciale. Elle constitue également un enjeu démographique pour les leaders de la communauté anglophone de Québec qui souhaitent voir ces jeunes s'intégrer en français au Québec et qui s'inquiètent de leur départ à l'extérieur de la province lorsque vient le temps de poursuivre des études universitaires ou de s’intégrer professionnellement.

\section{Bibliographie}

Arnett, J. J. (2007). Emerging Adulthood in Europe: A Response to Bynner. Journal of Youth Studies, 9(1), 111-123.

Bertaux, D. (2005). Le récit de vie, $2^{\mathrm{e}}$ édition. Paris, France : Armand Colin.

Corbeil, J.-P., Grenier, C. et Lafrenière, S. (2007). Les minorités prennent la parole : résultats de l'Enquête sur la vitalité des minorités de langue officielle, n 91-548-XIF au catalogue. Ottawa, Ontario : Ministre de l'Industrie.

Corcuff, P. (1995). Les nouvelles sociologies : constructions de la réalité sociale, F. de Singly (Ed.). Paris, France : Nathan.

Dallaire, C. (2008). La reproduction de l'identité francophone chez les jeunes : Le rôle de la stabilité des discours identitaires et de la représentation de la culture. Francophonies d'Amérique, 26, 357-381.

Darmon, M. (2006). La socialisation. Paris, France : Armand Colin.

Dubar, C. (2010). La socialisation (4 édition). Paris, France : Armand Colin.

Dubar, C. (1996). La socialisation. Construction des identités sociales et professionnelles. Paris, France : Armand Colin.

Dubet, F. (2002). Le déclin de l'institution. Paris, France : Seuil. 
Dubet, F. (1994). Sociologie de l'expérience. Paris, France : Seuil.

Duru-Bellat, M. et Van Zanten, A. (2006). Sociologie de l'école ( $3^{\mathrm{e}}$ édition). Paris, France : Armand Colin.

Farmer, D. et Heller, M. (2008). La sociologie de l'éducation. In J. Lafontant et S. Laflamme (Eds.) Initiation thématique à la sociologie (pp. 121-156). Sudbury, Ontario : Éditions prise de parole.

Galland, O. (1990). Un nouvel âge de la vie. Revue française de sociologie, XXXI, 529551.

Gérin-Lajoie, D. (2011). Youth, language and identity: portraits of students from English-language high schools in the Montreal area. Toronto, Ontario : Canadian Scholars' Press In.

Giddens, A. (1991). Modernity and self-identity. Self and society in the late modern age. Stanford, CA : Stanford University Press.

Jedwab, J. (2002). The Chambers report, ten years after: the state of English language education in Quebec, 1992-2002. Montréal, Québec : The Missisquoi Institute.

Kaufmann, J.-C. (2004). L’invention de soi : une théorie de l’identité. Paris, France : Hachette littératures.

Lamarre, P. (2005). L’enseignement du français dans le réseau scolaire anglophone : à la recherche du bilinguisme. In A. Stefanescu et P. Georgeault (Eds.) Le français au Québec : les nouveaux défis (pp. 553-568). Montréal, Québec : Éditions Fides.

Lamoureux, S. (2005). Transition scolaire et changements identitaires. Francophonies d'Amérique, 2O, 111-121.

Landry, R., Allard, R. et Deveau, K. (2010). École et autonomie culturelle. Enquête pancanadienne en milieu scolaire francophone minoritaire. Nouvelles perspectives canadiennes, Patrimoine Canadien.

Landry, R., Deveau, K. et Allard, R. (2006). Vitalité ethnolinguistique et construction identitaire : le cas de l'identité bilingue. Éducation et francophonie, XXXIV(1), 54-81.

Lipiansky, E. M. (1998). Comment se forme l'identité des groupes. In J.-C. RuanoBorbalan (ed.), L’identité : L’individu, le groupe, la société (pp.143-150). Auxerre et Paris, France : Science humaines et P.U.F.

Magnan, M.-O. (2010). Franchir les frontières scolaires, franchir les frontières identitaires ? De l'école anglaise vers l'université francophone au Québec. Ethnologies, 31(2), 289-312.

Martel, A. (2001). Droits, écoles et communautés en milieu minoritaire, 19862002 : analyse pour un aménagement du français par l'éducation. Ottawa, Ontario : Commissariat aux langues officielles.

Molgat, M. (2007). Do transitions and social structures matter? How 'emerging adults' define themselves as adults? Journal of Youth Studies, 10(5), 495-516.

Molgat, M. et Vézina, M. (2008). Transitionless biographies? Youth and representations of solo living. Young, 16(4), 349-371. 
Perret-Clermont, A.-N. et Zittoun, T. (2002). Esquisse d'une psychologie de la transition. Éducation Permanente, 1, 12-15.

Pilote, A. (2007). Suivre la trace ou faire son chemin? L'identité culturelle des jeunes en milieu francophone hors Québec. Revue internationale d'études canadiennes, 36, 229-251.

Pilote, A. et Bolduc, S. (2007). L'école de langue anglaise au Québec. Bilan des connaissances et nouveaux enjeux - Document d'interprétation rétrospectif (phase 1). Moncton, Nouveau-Brunswick : Institut canadien de recherche sur les minorités linguistiques.

Pilote, A. et Magnan, M.-O. (2012). Regards croisés sur les parcours objectifs et subjectifs à l'enseignement supérieur : une analyse diachronique de la construction identitaire des jeunes issus d'un contexte linguistique minoritaire au Canada. In F. Picard et J. Masdonati (Eds.) Les parcours d'orientation des jeunes : dynamiques institutionnelles et identitaires (pp. 225-254). Québec, Québec : Presses de l'Université Laval.

Pilote, A. et Magnan, M.-O. (2008). L'école de la minorité francophone au Canada : l'institution à l'épreuve des acteurs. In J.-Y. Thériault, A. Gilbert et L. Cardinal (Eds), L'espace francophone en milieu minoritaire au Canada : nouveaux enjeux, nouvelles mobilisations (pp. 275-317). Montréal, Québec : Fides.

Pilote, A., Magnan, M.-O. et Vieux-Fort, K. (2010). L'identité et le poids des langues : une étude comparative entre des jeunes de milieu scolaire francophone au NouveauBrunswick et anglophone au Québec. Nouvelles perspectives en sciences sociales : revue internationale de systémique complexe et d'études relationnelles, 6(1), 65-98.

Statistique Canada (2007). Le portrait linguistique en évolution, Recensement de 2006, N 97-555-XIF au catalogue. Repéré à http://www12.statcan.ca/francais/censuso6/ analysis/language/pdf/97-555-XIF2006001.pdf.

Stevenson, G. (1999). Community besieged. The Anglophone minority and the politics of Quebec. Montréal, Québec et Kingston, Ontario : McGill-Queen's University Press.

Strauss, A. L. (1997). Mirrors and masks: the search of identity. New Brunswick, NJ : Transaction Publishers.

Thériault, J.-Y. (2002). De l'école de la nation aux écoles communautaires ou de l'école d'en-haut à l'école d'en-bas. In Actes du colloque pancanadien sur la recherche en éducation en milieu francophone minoritaire. Moncton, Nouveau-Brunswick : CRDE et ACELF.

Vieux-Fort, K. et Pilote, A. (2010). Représentations de la communauté anglophone et positionnements identitaires chez des jeunes scolarisés en anglais à Québec : explorations méthodologiques. Glottopol, Revue de sociolinguistique en ligne, 16, 81-99.

Wiborg, A. (2001). Education, mobility and ambivalence. Rural students in higher education. Young: Nordic Journal of Youth Research, 9(1), 23-40. 


\section{Notes}

1. La clause Canada, c'est-à-dire répondre au critère " avoir un parent ayant reçu la majorité de son enseignement primaire en anglais au Canada » pour être admissible à l'école de langue anglaise, est adoptée en 1984 par un arrêt de la Cour Suprême du Canada. Auparavant, la clause Québec prévalait, c'est-à-dire répondre au critère « avoir un parent ayant reçu la majorité de son enseignement primaire en anglais au Québec » pour être admissible à l'école de langue anglaise.

2. En raison de cette homogénéité sur le plan du statut socioéconomique des parents des jeunes interrogés, nous n'avons pu effectuer d'analyse en fonction de cette variable.

3. Des pseudonymes sont utilisés tout au long de l'article afin de respecter la confidentialité de l’identité des répondants.

\section{Coordonnées}

Marie-Odile Magnan

Professeure adjointe

Département d'administration et fondements de l'éducation

Université de Montréal

2900 Boulevard Edouard-Montpetit

Montreal, QC H3T 1J4 Canada

marie-odile.magnan@umontreal.ca

Marie-Odile Magnan est professeure adjointe au Département d'administration et fondements de l'éducation de l'Université de Montréal. Elle possède un doctorat en sociologie de l'Université Laval. Elle est chercheure au Centre d'études ethniques des universités montréalaises (CEETUM) et à l'Observatoire Jeunes et Société (INRS UCS). Outre la sociologie de l'éducation, ses intérêts de recherche portent sur l'analyse des enjeux culturels, linguistiques et ethniques en éducation. Elle s'intéresse plus spécifiquement à la fonction linguistique et culturelle des institutions scolaires et à la construction identitaire chez les jeunes de minorités linguistiques et ethniques au Canada. 